\title{
Combined Structural and Synovial Assessment for Improved Ultrasound Discrimination of Rheumatoid, Osteoarthritic, and Normal Joints: A Pilot Study
}

\author{
Gary A. Kunkel*, Grant W. Cannon and Daniel O. Clegg
}

George E. Wahlen VA Medical Center, Division of Rheumatology, University of Utah School of Medicine, USA

\begin{abstract}
Background: Current ultrasonographic scoring systems used to assess the degree of finger joint synovitis in rheumatoid arthritis (RA) are not designed for distinguishing healthy or osteoarthritis (OA) patients from those with RA in clinical settings.

Objective: To explore a novel scoring approach using structural and synovial ultrasonographic features to distinguish between healthy and OA finger joints and those with RA.

Methods: 22 patients with RA, 16 healthy controls, and 14 OA controls received a comprehensive ultrasound of one hand, with scores assigned using a modification of a previously reported RA scoring system (Semiquantitative Synovial Score), and using the novel approach (Combined Structural/Synovial Score). The number of joints classified as supporting the diagnosis of RA ("RA-supported") with each approach was recorded. Sensitivity and specificity for each scoring system were calculated with respect to the clinical diagnosis.

Results: The Semiquantitative Synovial Score was highly sensitive (100\%), but without specificity ( $0 \%)$ for the diagnosis of RA, when RA was defined as having more than 1 joint classified as "RA-supported." The Combined Structural/Synovial Score had high sensitivity (95\%) and moderate specificity (77\%) when RA was defined as having any joint classified as "RA-supported". Moderate sensitivity (73\%) and high specificity (97\%) were found when having more than 1 joint classified as "RA-supported" was required to diagnose RA.

Conclusion: A novel structural and synovial hand joint scoring system was capable of distinguishing OA and healthy controls from RA subjects in this pilot evaluation. Prospective validation of this approach is planned.
\end{abstract}

Keywords: Ultrasound, scoring system, rheumatoid arthritis, osteoarthritis.

\section{INTRODUCTION}

Musculoskeletal ultrasound (MskUS) is a tool increasingly found to be of value in rheumatology clinics in many parts of the world [1-4]. Two common indications for the use of MskUS are to assist in the clinical diagnosis of inflammatory arthritis and to document and quantify the degree of synovitis. The Outcome Measures in Rheumatology network (OMERACT) definitions for ultrasound pathology [5] provide a consensus for defining inflammatory changes, but have not been recommended as a clinical tool for use in diagnosing or assessing inflammatory arthritis. In general, two semiquantitative classification systems for grayscale MskUS evaluation of metacarpophalangeal (MCP) and proximal interphalangeal (PIP) joint synovitis have been studied. One approach emphasizes qualitative assessments of the location of visible synovial tissue [6]. The second method grades detectable synovial tissue or fluid as: "absent"; "minimal"; "moderate"; or "extensive" [7]. Similarly, two semiquantitative scoring systems for Doppler examination are commonly reported,

*Address correspondence to this author at the George E. Wahlen VAMC, 500 Foothill Drive (11E), Salt Lake City, UT 84148, USA; Tel: (801)581-4333; Fax: (801)581-6069; E-mail: Gary.kunkel@hsc.utah.edu the first grades Doppler findings as "none", "mild", "moderate", or "marked" [8], and the second assigns a descriptive grade that includes the proportion of synovium with visible Doppler signals [9]. These scoring systems, individually and in combination, have been correlated with synovial pathological specimens and MRI findings, and shown to be more sensitive than clinical joint evaluation. They have also been shown to predict the development of RA in undifferentiated synovitis, to detect response to change in RA therapy, and predict future joint damage [6, 7, 9-17]. Despite these promising results, and the utility of such scoring systems in the clinical trial setting, the clinical applicability of these scoring systems in distinguishing osteoarthritis or normal patients from RA patients has not been clearly demonstrated.

In clinical trials, the question is defining the degree of synovitis in patients with RA, while in clinical practice the question is often determining whether or not the patient has RA. Thus there is a real need for an MskUS scoring system to successfully distinguish a normal joint from a pathological joint, and an inflammatory arthropathy from a degenerative arthropathy. One currently used [6] scoring system has been tested against normal controls with mixed results $[12,18$, 19], perhaps due to differences in scanning planes used. Using a modified semiquantitative score, Scheel et al. found 
good sensitivity and specificity when RA patients were compared to healthy controls [7]. None of the currently available scoring systems for RA synovial disease have been evaluated using osteoarthritis (OA) joints as controls. Given recent studies of MskUS in hand OA, it is likely that a large number of elevated synovial disease scores would be identified using these scoring systems in OA [20-25]. Finally and of concern in clinical practice, is the subjective nature of semiquantitative scores, which could easily lead to misdiagnosis of inflammatory arthritis in the clinical setting, particularly among less experienced ultrasonographers.

This report describes an exploration of a novel clinical scoring system of hand arthritis, using a combined structural/qualitative/quantitative approach. The design of the combined structural and synovial scoring system incorporates published normal values for synovial cavity volume of the MCP and PIP joints [26], anatomical descriptions of the positions of synovial fluid and synovial proliferation, Doppler signal, and the easily visible structural detail of osteophytes and erosions.

\section{MATERIALS AND METHODOLOGY}

\section{Subjects}

Twenty-two patients fulfilling 1987 ACR criteria for RA [27], 14 patients with clinical and radiographic hand OA (6 of whom had radiographic findings of erosive osteoarthritis), and 16 healthy volunteers with no hand complaints or systemic arthritis were recruited for participation. The recruited patients were from either the rheumatology clinic at the University of Utah Health Sciences Center or the George Wahlen Veterans Administration Medical Center, with some of the healthy controls coming from staff at these institutions as well. The study was performed with the approval and supervision of the local institutional review board. All patients and healthy controls gave signed informed consent to participate in the study.

\section{Ultrasound Exam}

Ultrasound examination of one hand per patient was performed using either a Siemens Acuson X300 with a 5-13 $\mathrm{MHz}$ linear transducer (Siemens, WA, USA), or a Biosound Esaote MyLab 25 with a 10-15 MHz linear transducer (Biosound Esaote, CA, USA), the use of the machine depended on the hospital at which the exam was performed.

All examinations were performed by a single investigator, with 5 years of experience in musculoskeletal ultrasonography (GAK). The ultrasonographer was not systematically aware of the diagnoses of the subjects, but did have clinical familiarity with in particular many of the Veteran's population diagnoses. Each examination took approximately 30-45 minutes (actual scanning time 10-15 minutes), and included grayscale and Doppler imaging of each MCP and PIP joint (Doppler settings of low wall filter, frequency 7.3 or $8 \mathrm{MHz}$, pulse repetition frequency of 500 or $600 \mathrm{~Hz}$, with gain set just below the limit of visible subcortical noise). Given the frequent occurrence of synovial tissue or fluid previously described in oblique planes [18], a longitudinal circumferential approach was used for scanning each MCP and PIP, to include as much radial and ulnar imaging of the joint as possible, along with standard midsagittal images, and short axis imaging of any visualized cortical disruptions. Doppler, synovial, erosive and productive changes were recorded at the time of scanning for utilization in the scoring systems as described below.

\section{Ultrasound Scoring Systems}

\section{Semiquantitative Synovial Score}

This is a scoring system based on previously published protocol [6] that has been extensively studied and correlated with MRI in normal controls and RA patients [12, 18, 19]. Scores as defined in Table 1 were recorded for each joint, and the total number of joints with scores supporting the diagnosis of RA ("RA-supported") in each patient recorded.

Some differences in the Semiquantitative Synovial Score from the original description of this scoring system should be noted. As the exact position of scanning (i.e. dorsal, dorsal-radial, dorsal-ulnar, etc...) has not been described in the previously reported scoring system, the highest score seen in any scanning plane was assigned to the joint. The value of measuring synovial tissue as a separate parameter from synovial fluid is not clear, and it is not always easy to visualize the separation of the fluid from synovium. Therefore these were combined in a single measure in this report as has been done by others [7, 18]. Due to the variability in echogenicity of synovial tissue as well as the dorsal homogenous connective tissue that is intracapsular in the MCP and PIP joints (sometimes loosely termed a "fat pad"), a dorsal score was only assigned if the hypoechoic synovial tissue was distinguishable from the intracapsular connective tissue, or was markedly hypoechoic at multiple angles of insonation. A score was only assigned volarly if the hypoechoic tissue was distinguishable from the homogenous, hyperechoic palmar plate and surrounding connective tissue or muscle. The highest score as defined dorsally or volarly was assigned to an individual MCP or PIP joint. In the original report of this scoring system, a score of 3 or 4 is defined as visible synovium along the diaphysis that occurs in the setting of visible bulging over the line drawn between the joint-forming bones (in other words a score of 3 or 4 assumes that a score of 2 is already present). No specific mention is made of how to score a joint where visible

\section{Table 1. Definitions of Grade 0-4 Scores for the Semiquantitative Synovial Score}

\begin{tabular}{|l|l|}
\hline Grade 0 & No Visible Hypoechoic Tissue \\
\hline Grade 1 & Hypoechoic thickening between the bones that form a joint, not exceeding a line drawn between the tops of the bones \\
\hline Grade 2 & $\begin{array}{l}\text { Hypoechoic thickening visible above the line drawn between the tops of the bones that form a joint, but that does not extend to overlie the } \\
\text { diaphysis of either bone }\end{array}$ \\
\hline Grade 3 & Hypoechoic thickening that extends to overlie either the proximal or distal diaphysis of one of the bones \\
\hline Grade 4 & Hypoechoic thickening that extends to overlie both the proximal and distal diaphysis of the bones \\
\hline
\end{tabular}


synovium is seen along the diaphysis (which suggests a score of 3 or 4), but is not seen to bulge above the line between the joint-forming bones. We elected to score such joints as a 3 or 4 . Given the modifications described above which would be expected to increase sensitivity and decrease specificity, a higher score of 3 or 4 was required for a joint to be identified as supporting the diagnosis of RA("RAsupported") in this system, whereas a score of 2 was defined as abnormal in the initial description.

\section{Combined Structural/Synovial Score}

This is a novel scoring system which was developed utilizing a combination of synovial and bony structural parameters in an attempt to better differentiate RA patients from $\mathrm{OA}$ and normal controls. This system was defined so that each MCP or PIP joint is evaluated independently and can be classified as "RA-supported" or "RA-unsupported", depending on the findings. Classifying a joint as "RA-supported" is equivalent to stating "the bony, Doppler, and/or synovial findings in this joint are suggestive of rheumatoid arthritis". This system is outlined as a flow diagram in Fig. (1).

Step 1. The presence or absence of an osteophyte is determined. Any abnormal bony prominence at the joint line is defined as an osteophyte, and an osteophyte leads to automatic classification of the joint as "RA-unsupported". The next joint may be assessed without further review.

Step 2. When no clear osteophytes are present, the presence or absence of erosions and Doppler signal are established. A joint is defined as having an erosion in this series if a cortical breach greater than $1 \mathrm{~mm}$ in width is visible in two orthogonal planes. Doppler scores were assigned semiquantitatively 0 to 3 as has been previously defined [9]. Any erosion or Doppler signal greater than $1+$ establishes a joint as "RA-supported."

Step 3. Finally, the presence or absence of abnormal synovial tissue or fluid is established. Visible synovial tissue/fluid is defined as anechoic fluid or hypoechoic tissue, distinguishable from the normal, homogenous dorsal intracapsular tissue ("fat pad") or volar palmar plate in each joint, and seen in any scanning plane. To classify a joint as "RAsupported", this hypoechoic tissue or anechoic fluid must be visible along the proximal or distal diaphysis of either joint-forming bone, corresponding to a score of at least 3 in the Semiquantitative Synovial Score. Given the large number of normal patients that have such visible synovium/fluid in at least one scanning plane [18], a quantitative cutoff of $>2 \mathrm{~mm}$ of visible synovium/fluid as measured perpendicularly anywhere from the diaphyseal surface (or, if they synovium/fluid was not apposed directly to the bone, in a direction perpendicular to the long axis of the digit) was used to classify the joint as "RAsupported". This number is based on previously determined normal control values for MCP and PIP

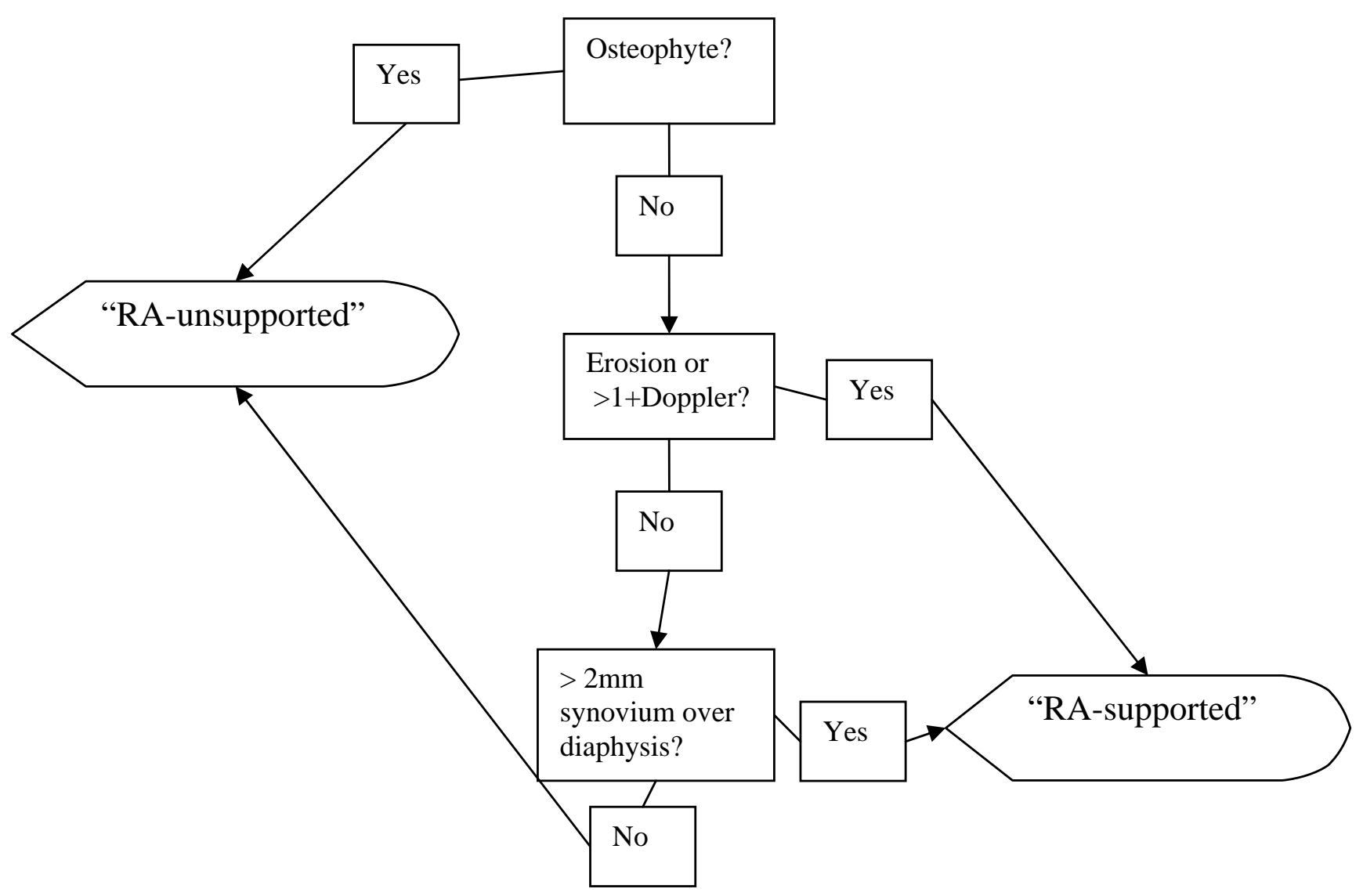

Fig. (1). Flow diagram for the classification of a joint in the Combined Structural/Synovial Score. 
joints [26]. If both the proximal and distal bones have overlying synovial tissue/fluid (i.e. a score of 4 in the Semiquantitative Synovial Score), the greater of the two synovial distensions is measured, in practice this was almost always the proximal measurement (data not shown). Ultrasound images of MCP and PIP joints and related commentary on the use of both scoring systems are shown in Figs. $(2,3)$, respectively.

\section{Data Analysis}

Sensitivity for the diagnosis of RA, and specificity when compared to osteoarthritic, healthy, and all control subjects were calculated for the two scoring systems using different cutoffs for the two systems. Receiver operating characteristic (ROC) curves were plotted for the two scoring systems using all available cutoff points.

\section{RESULTS}

The baseline demographic, disease characteristics, and selected US findings of the study population are shown in Table 2. The RA population reflected the demographics of the Intermountain West veteran population; OA patients were recruited from both the University and Veteran's clinics.

A comparison of the Semiquantitative Synovial Score and the Combined Structural/Synovial Score is shown in
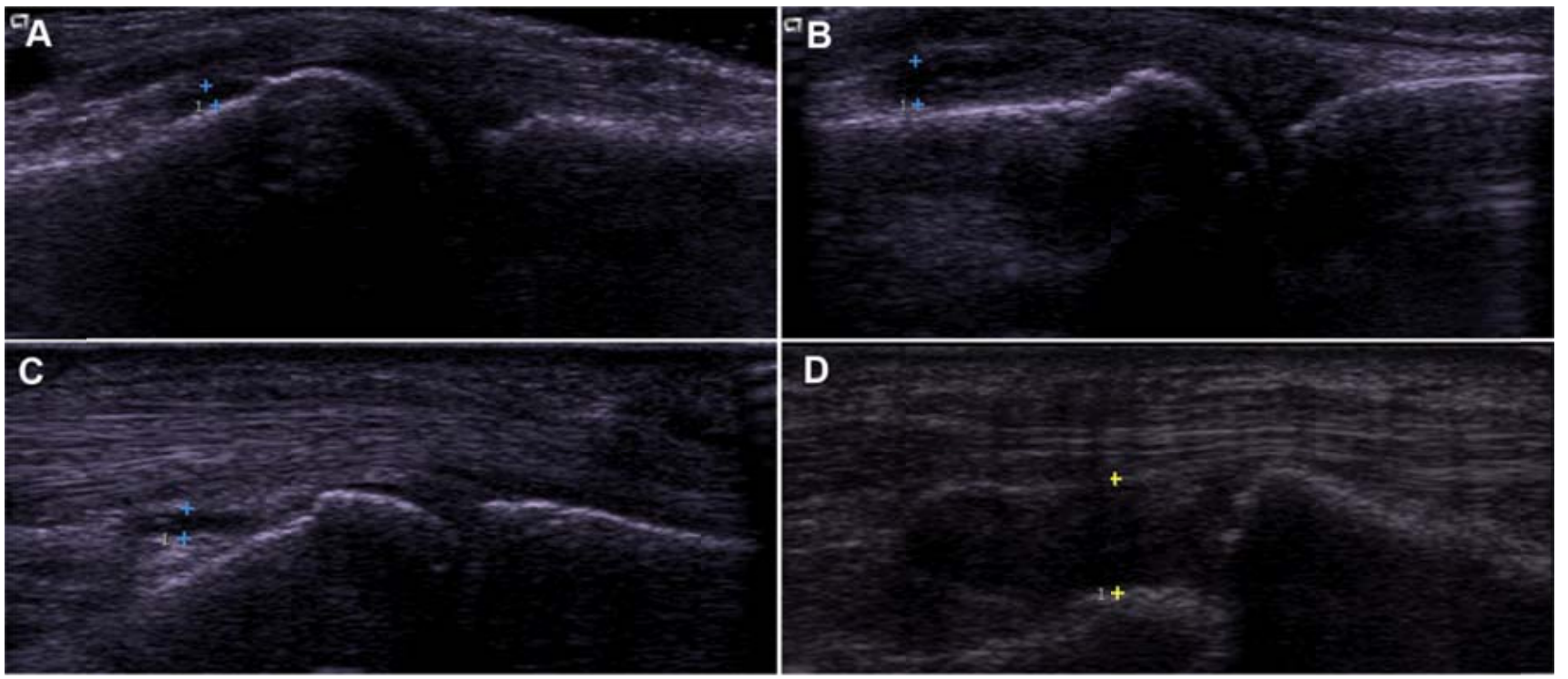

Fig. (2). Dorsal (A, B) and volar (C, D) scans of MCP joints. All four of these joints were assigned a score of 3("RA-supported") using the Semiquantitative Synovial Score, while A and C were classified as "RA-unsupported" using the Combined Structural/Synovial Score. Proximal is to the left in all images.
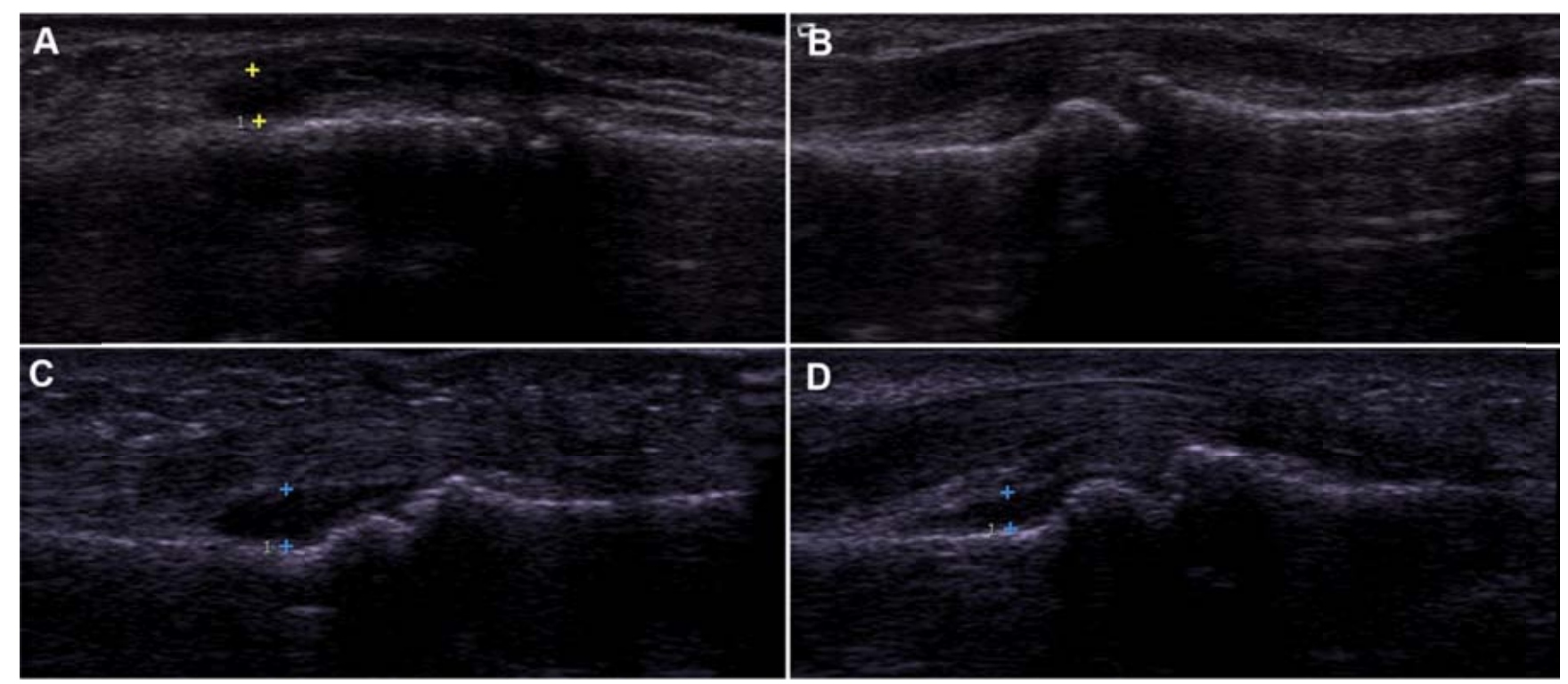

Fig. (3). Varying degrees of synovial cavity distension in dorsal (A) and volar (B-D) scans of PIP joints. All 4 images were given a grade of 3("RA-supported") using the Semiquantitative Synovial Score, while images B and D were classified as "RA-unsupported" using the Combined Structural/Synovial Score. 
Table 2. Demographic and Clinical Characteristics of the Study Population

\begin{tabular}{|c|c|c|c|}
\hline Clinical Characteristics & Rheumatoid Arthritis $(\mathbf{n}=\mathbf{2 2})$ & Osteoarthritis Control (n=14) & Healthy Control (n=16) \\
\hline \hline Median Age & 60 & 69 & 35 \\
\hline Proportion female & 0.27 & 0.86 & 0.19 \\
\hline Proportion caucasian & 0.95 & 1.0 & 0.94 \\
\hline Proportion CCP + & 0.65 & N/A & N/A \\
\hline Average DAS at evaluation* & 3.74 & 0.50 & N/A \\
\hline Proportion with any US synovial proliferation $>2 \mathrm{~mm}$ & 0.86 & 1.0 & 0.06 \\
\hline Proportion with any US osteophyte+ & 0.23 & 0.12 \\
\hline
\end{tabular}

*DAS values were available within a week of the day of the evaluation for $68 \%$ of the RA population

+ Defined as an abnormal bony prominence at or near the joint margin.

Table 3. Sensitivity (Sensitivity-RA) and Specificity for the Diagnosis of RA when Compared to Osteoarthritis (Specificity-O), Healthy (Specificity-H), and All Controls (Specificity-All) Using the Semiquantitative Synovial Score and the Combined Structural/Synovial Score

\begin{tabular}{|c|c|c|}
\hline "RA-Supported" $>\mathbf{0}$ & Semiquantitative Synovial Score & Combined Structural/Synovial Score \\
\hline \hline Sensitivity-RA & 1 & 0.95 \\
\hline Specificity-O & 0 & 0.57 \\
\hline Specificity-H & 0 & 0.94 \\
\hline Specificity-All & 0 & Combined Structural/Synovial Score \\
\hline "RA-supported" $>\mathbf{1}$ & Semiquantitative Synovial Score & 0.73 \\
\hline \hline Sensitivity-RA & 1 & 0.93 \\
\hline Specificity-O & 0 & 1 \\
\hline Specificity-H & 0 & 0.97 \\
\hline Specificity-All & 0 & 0.77 \\
\hline
\end{tabular}

The top half of the table shows results when a patient is diagnosed as RA if any joint is classified as "RA-supported", and the bottom half shows results when a patient is diagnosed as RA if more than one joint is classified as "RA-supported".

Table 3. Sensitivities and specificities of the two scoring systems are shown when a subject is assigned the diagnosis of RA if $>0$ or $>1$ joint is classified as "RA-supported" using either scoring system. The Semiquantitative Synovial Score lacked specificity in distinguishing the controls from the RA patients. The Combined Structural/Synovial Score yields fair specificity, with excellent sensitivity when a subject is diagnosed as RA if more than 0 joints are classified as "RAsupported", and yields good sensitivity, with excellent specificity, if more than 1 "RA-supported" joint is required to assign the diagnosis of RA. To further evaluate the two scoring systems, ROC curves were plotted using the complete range of possible scores against tests with ideal or useless operating characteristics. These results are shown in Fig. (4).

Results of a sensitivity analysis of several different variations of the Combined Structural/Synovial Score reveal important differences in the sensitivity and specificity of this system when different parameters are used. For example, ignoring the first digit does not change the sensitivity and specificity of the Combined Structural/Synovial Score if finding $>1$ "RA-supported" joint is used for assigning the diagnosis of RA, but leads to less sensitivity if $>0$ "RA- supported" joints is used for diagnosing RA. Using a lower cutoff for PIP joint synovial cavity distension to be defined as "RA supported" (1.5 $\mathrm{mm}$ instead of $2 \mathrm{~mm}$ ) leads to a slightly higher sensitivity of the Combined Structural/Synovial Score for the diagnosis of RA, but a significantly lower specificity. In this case, increased numbers of both normal and OA controls were identified as "RA". The Combined Structural/Synovial Score was also separately examined when only dorsal or volar evaluations were included. Using only dorsal evaluation yielded a sensitivity slightly lower than when using both dorsal/volar scans, with similar specificity. Using only volar scans led to a significantly diminished sensitivity when compared to using both dorsal/volar scans or only dorsal scans.

\section{DISCUSSION}

Currently available MskUS scoring systems are effective in assessing the activity of RA synovitis and predicting disease progression. However, the clinical utility of current scoring systems for the diagnosis of RA is less clear, and clinical experience as well as prior study suggests the potential for overdiagnosis of pathology if currently available systems are used for diagnostic purposes in Rheumatology practices. In this study, a novel scoring 


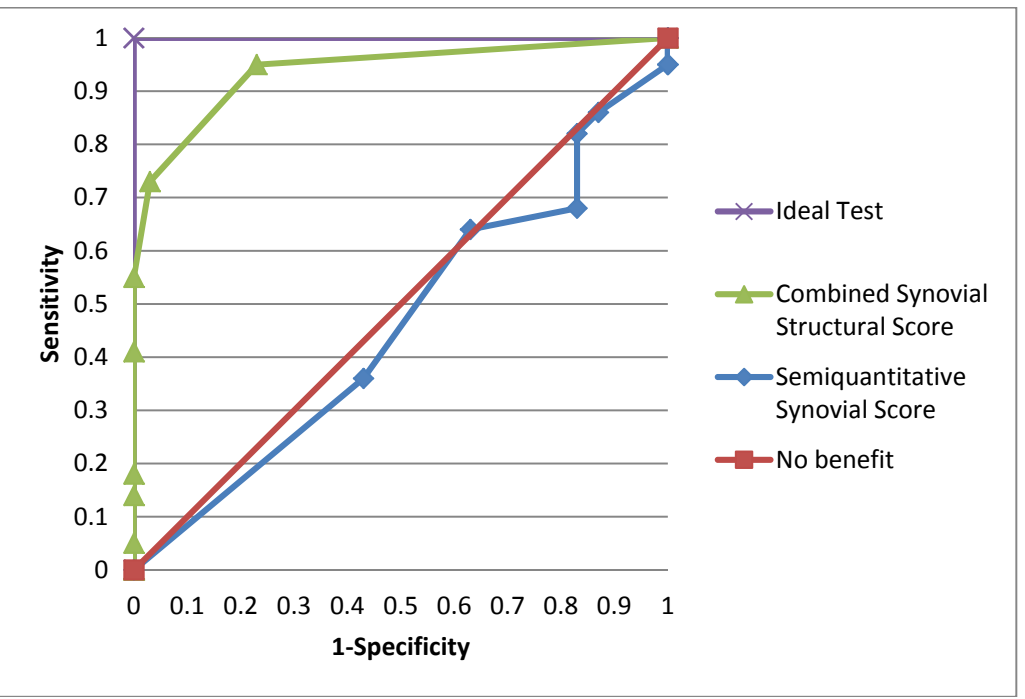

Fig. (4). Receiver operating characteristic curves for the Combined Synovial Structural and the Semiquantitative Synovial Scores plotted against hypothetical tests with no benefit or ideal operating characteristics over the range of possible scores from 0 to 10 .

system that combines structural and synovial changes in individual MCP and PIP joints to distinguish RA patients from $\mathrm{OA}$ patient controls and healthy controls performed with good sensitivity and specificity, compared to a semiquantitative scoring system without structural parameters.

In agreement with Ellegaard et al. [18] we found a high occurrence of grade 2 or higher synovial scores according to the Semiquantitative Synovial Score when evaluating control joints in multiple scanning planes, which led to a lack of specificity of the Semiquantitative Synovial Score for the diagnosis of RA. This is in contrast to the original use of this system by Szkudlarek et al. [12] and subsequent use of a version of this scoring system by Wiell et al. [28] and Millot et al. [19] where less frequent abnormal scores were seen in healthy controls. Much of this difference is likely attributable to the use of multiple scanning planes, which may allow detection of synovial tissue in the previously described "tails" of the joint recess that may be more prominent medially or laterally to the midline [29]. In many cases, we found proximal synovium/fluid collections over the diaphysis (score of 3) using the Semiquantitative Synovial Score, in the absence of concomitant bulging over the lines between the joint-forming bones (score of 2). It may be that for further precision in the Semiquantitative Synovial Score, explicit rejection of scoring fluid/synovium overlying the diaphysis should be emphasized when the bulging that defines a score of 2 is not also seen. However, on the volar surface, where in our and others [18] experience a score of 2 is almost never seen due to the pressure of the volar plates on the joint line, this would have to be further clarified. Lastly, because of uncertain utility, and given difficulty in distinguishing synovial proliferation from effusion with varying equipment, both effusion and proliferation were scored together, which increases the detection of "abnormal" distension in the Semiquantitative Synovial Score when compared to the original description, in which only synovial tissue was scored in this way.

A cutoff of $2 \mathrm{~mm}$ was used to define the amount of synovium or fluid as "RA-supported" for both MCP and PIP joints, an upper limit of normal that exceeded 2 standard deviations above normal values as described by Schmidt et al. [26] in their study of 102 healthy volunteers $(1.9 \mathrm{~mm}$ and $1.6 \mathrm{~mm}$ for MCP and PIP joints, respectively). This cutoff yielded acceptable sensitivity and specificity, but differs substantially from the study by Scheel et al. [7], which found the optimum cutoff to be $0.6 \mathrm{~mm}$ for both MCP and PIP joints, despite similar measurement techniques. In our patient population, using a cutoff of $0.6 \mathrm{~mm}$ would have led to the assignment of several joints in each healthy and many joints in each OA control patient as "RA-supported" (data not shown). The reasons for this difference are not entirely clear, but may somewhat be related to our use of both dorsal and volar evaluations, as well as multiple scanning planes as outlined in the methods. Each of these differences increases the likelihood of finding synovium/fluid overlying the diaphysis. Some disagreement exists in the literature on the necessity of examining both dorsal and volar planes of the finger joints $[6,7]$. In our study population more extensive synovial proliferation and fluid were identified when dorsal rather than volar scans were used, in particular at the MCP joints, but the highest sensitivity was seen when both dorsal and volar scans were included.

As expected from previous studies [20-25], high numbers of joints with synovial proliferation/effusion were identified in the OA controls. Half of the OA control patients had at least one joint with more than $2 \mathrm{~mm}$ of synovial distension, and half had at least one joint with $1+$ Doppler signal, with one patient with erosive OA having $2+$ Doppler signal in several joints. Thiele et al. have recently described differing characteristics of synovial distension in OA and RA, noting that osteophytes can mechanically distend the joint capsule [30]. This distension by osteophytes can be thought of as a "tent pole" propping up the canvas (synovium), and may promote filling of the "tent" space by joint fluid (see Fig. 5). In addition, low grade inflammation is often present in OA joints, in particular patients with erosive osteoarthritis, which may lead to a distended synovial cavity without necessarily relying on this "tent pole" phenomenon. Furthermore, erosive $\mathrm{OA}$ is defined by erosions, and even in nonerosive OA the bony distortion caused by osteophytes may make the determination of erosive disease difficult using US. In 

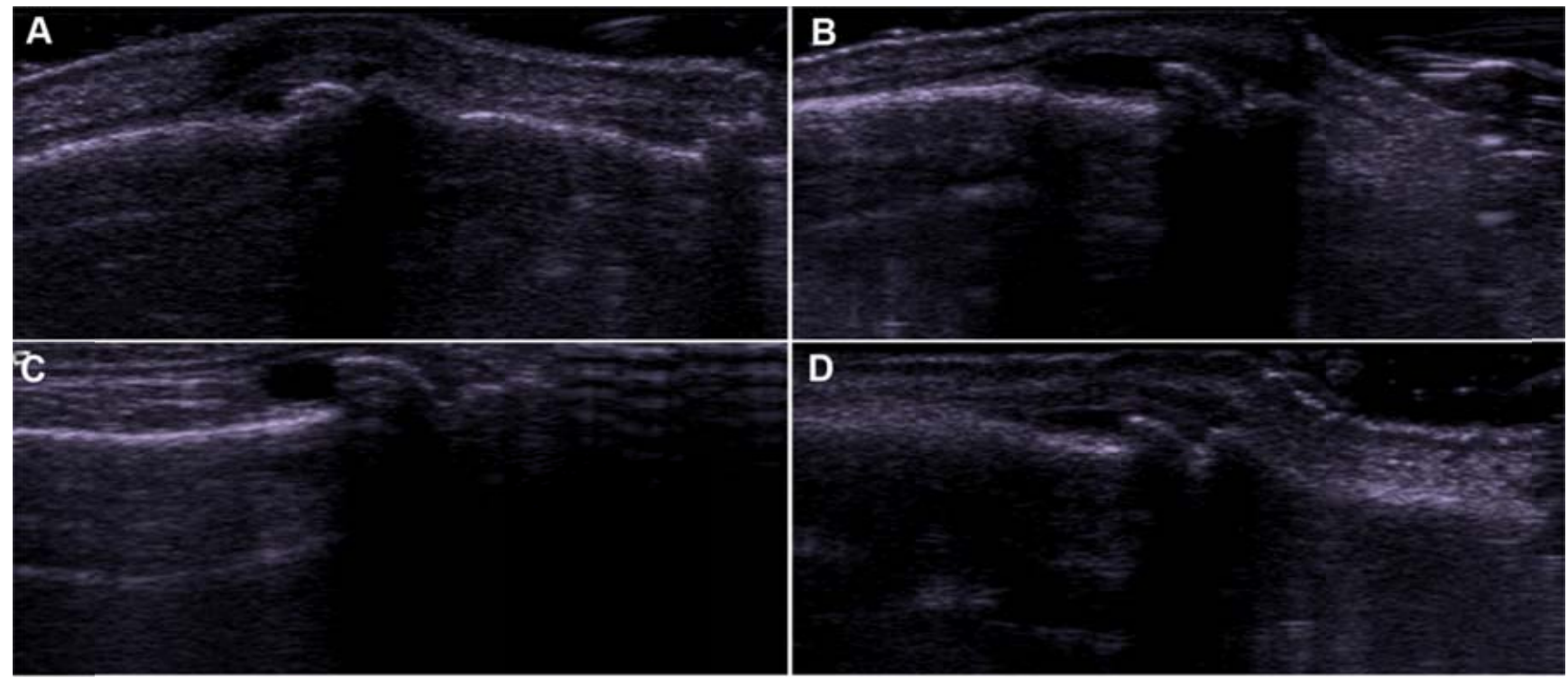

Fig. (5). PIP joint "tent poles" showing distended synovial cavities along the height of dorsal osteophytes. All 4 images were graded as a 3 ("RA-supported") using the Semiquantitative Synovial Score, while the presence of osteophytes gave an "RA-unsupported" classification for each using the Combined Structural/Synovial Score.

addition, recent study has shown that bony marginal erosions may be identified even in patients with "nonerosive" OA on careful radiographic inspection, though these are much more commonly seen in the DIP than PIP joints, and strongly correlated with the presence of osteophytes [31]. These factors justify disallowing an "RA-supported" score for a joint which has erosions, if an osteophyte is also present.

Key limitations in this study and approach should be emphasized. The veteran population and male Caucasian predominance of RA patients may limit the broader applicability of the findings. In addition, the healthy controls, while also being predominantly male, were significantly younger than the patients, and it is possible that age-matched (older) control subjects may have had a higher prevalence of subclinical joint pathology. Lack of effective blinding in many of the patients is also a limitation, but may be somewhat mitigated by the use of measured rather than more subjective parameters. This approach was useful in separating established RA patients from OA and normal patients, but would not be expected to distinguish between different types of inflammatory arthritis, and may be less effective in early inflammatory arthritis patients. The use of a structural parameter in the proposed approach to scoring prevents misclassification of OA joints as "RA-supported" by disallowing this classification if an osteophyte is observed. However, this might lower the sensitivity of this approach in diagnosing RA when widespread concomitant or secondary OA is present. In our patient set, while several RA patients had osteophytes, no RA patient was misclassified as not having RA due to their presence. It may be that using higher synovial or Doppler cutoffs in joints with osteophytes could allow them to be scored as "RA-supported". However, with the synovial cutoffs used here, disallowing these joints is necessary to maintain specificity of our scoring approach. Lastly, the bony proliferation of psoriatic arthritis may reduce "RA-supported" scores, and this diagnosis must always be considered when bony and synovial proliferation exist together.

\section{CONCLUSION}

The proposed scoring system uses an innovative approach to combine structural and synovial tissue findings during MskUS to accurately distinguish RA patients from healthy and OA controls. Existing RA scoring systems have proven very useful in monitoring RA and assessing response in clinical trials, but may lead to overdiagnosis if used clinically for diagnosis of RA. The use of a quantitative cutoff for what constitutes abnormal synovial cavity distension may eliminate some of the risk of overdiagnosing inflammatory changes, but may still lead to overdiagnosis of RA in joints with osteophytes unless these joints are excluded. A scoring system such as the one explored here, if validated in larger, prospective, multireader studies, may have a diagnostic role in rheumatology clinic consultations. The time (in clinic) to complete the proposed scoring evaluation in any patient is expected to be from 10-15 minutes depending on the amount of synovial pathology present. Prospective validation with interreader comparisons is planned using a rheumatology intake clinic setting, and final diagnosis as the outcome variable.

\section{ACKNOWLEDGEMENTS}

The authors would like to thank the Western Institute for Biomedical Research for funding support for this project, and Mr. Russell Walston for his assistance in preparing this manuscript.

\section{CONFLICTS OF INTEREST}

None of the authors have any financial interests in this research which could create any real or apparent potential conflicts of interest. 


\section{REFERENCES}

[1] Wakefield RJ, Goh E, Conaghan PG, Karim Z, Emery P. Musculoskeletal ultrasonography in Europe: results of a rheumatologist-based survey at a EULAR meeting. Rheumatology (Oxford) 2003; 42: 1251-3.

[2] Takase K, Ohno S, Ideguchi H, Takeno M, Shirai A, Ishigatsubo Y. Use of musculoskeletal ultrasound in Japan: a survey of practicing rheumatologists. Mod Rheumatol 2010; 20: 376-80.

[3] Pineda C, Reginato AM, Flores V, et al. Pan-American League of Associations for Rheumatology (PANLAR) recommendations and guidelines for musculoskeletal ultrasound training in the Americas for rheumatologists. J Clin Rheumatol 2010; 16: 113-8.

[4] Samuels J, Abramson SB, Kaeley GS. The use of musculoskeletal ultrasound by rheumatologists in the United States. Bull NYU Hosp Jt Dis 2010; 68: 292-8.

[5] Wakefield RJ, D'Agostino MA, Iagnocco A, et al. The OMERACT Ultrasound Group: status of current activities and research directions. J Rheumatol 2007; 34: 848-51.

[6] Szkudlarek M, Court-Payen M, Jacobsen S, Klarlund M, Thomsen HS, Ostergaard M. Interobserver agreement in ultrasonography of the finger and toe joints in rheumatoid arthritis. Arthritis Rheum 2003; 48: 955-62.

[7] Scheel AK, Hermann KG, Kahler E, et al. A novel ultrasonographic synovitis scoring system suitable for analyzing finger joint inflammation in rheumatoid arthritis. Arthritis Rheum 2005; 52: 733-43.

[8] Newman JS, Laing TJ, McCarthy CJ, Adler RS. Power Doppler sonography of synovitis: assessment of therapeutic response-preliminary observations. Radiology 1996; 198: 582-4.

[9] Szkudlarek M, Court-Payen M, Strandberg C, Klarlund M, Klausen T, Ostergaard M. Contrast-enhanced power Doppler ultrasonography of the metacarpophalangeal joints in rheumatoid arthritis. Eur Radiol 2003; 13: 163-8.

[10] Koski JM, Saarakkala S, Helle M, Hakulinen U, Heikkinen JO, Hermunen H. Power Doppler ultrasonography and synovitis: correlating ultrasound imaging with histopathological findings and evaluating the performance of ultrasound equipments. Ann Rheum Dis 2006; 65: 1590-5.

[11] Rees JD, Pilcher J, Heron C, Kiely PD. A comparison of clinical vs ultrasound determined synovitis in rheumatoid arthritis utilizing gray-scale, power Doppler and the intravenous microbubble contrast agent 'Sono-Vue'. Rheumatology (Oxford) 2007; 46: 4549.

[12] Szkudlarek M, Klarlund M, Narvestad E, et al. Ultrasonography of the metacarpophalangeal and proximal interphalangeal joints in rheumatoid arthritis: a comparison with magnetic resonance imaging, conventional radiography and clinical examination. Arthritis Res Ther 2006; 8: R52.

[13] Ribbens C, Andre B, Marcelis S, et al. Rheumatoid hand joint synovitis: gray-scale and power Doppler US quantifications following anti-tumor necrosis factor-alpha treatment: pilot study. Radiology 2003; 229: 562-9.

[14] Naredo E, Collado P, Cruz A, et al. Longitudinal power Doppler ultrasonographic assessment of joint inflammatory activity in early rheumatoid arthritis: predictive value in disease activity and radiologic progression. Arthritis Rheum 2007; 57: 116-24.

[15] Boyesen P, Haavardsholm EA, van der Heijde D, et al. Prediction of MRI erosive progression: a comparison of modern imaging modalities in early rheumatoid arthritis patients. Ann Rheum Dis 2011; 70: 176-9.
[16] Naredo E, Moller I, Cruz A, Carmona L, Garrido J. Power Doppler ultrasonographic monitoring of response to anti-tumor necrosis factor therapy in patients with rheumatoid arthritis. Arthritis Rheum 2008; 58: 2248-56.

[17] Filer A, de Pablo P, Allen G, et al. Utility of ultrasound joint counts in the prediction of rheumatoid arthritis in patients with very early synovitis. Ann Rheum Dis 2011; 70(3): 500-7.

[18] Ellegaard K, Torp-Pedersen S, Holm CC, Danneskiold-Samsoe B, Bliddal $\mathrm{H}$. Ultrasound in finger joints: findings in normal subjects and pitfalls in the diagnosis of synovial disease. Ultraschall Med 2007; 28: 401-8.

[19] Millot F, Clavel G, Etchepare F, et al. Musculoskeletal Ultrasonography in Healthy Subjects and Ultrasound Criteria for Early Arthritis (The ESPOIR Cohort). J Rheumatol 2011; 38(4): 613-20.

[20] Keen HI, Lavie F, Wakefield RJ, et al. The development of a preliminary ultrasonographic scoring system for features of hand osteoarthritis. Ann Rheum Dis 2008; 67: 651-5.

[21] Keen HI, Wakefield RJ, Grainger AJ, Hensor EM, Emery P, Conaghan PG. An ultrasonographic study of osteoarthritis of the hand: synovitis and its relationship to structural pathology and symptoms. Arthritis Rheum 2008; 59: 1756-63.

[22] Vlychou M, Koutroumpas A, Malizos K, Sakkas LI. Ultrasonographic evidence of inflammation is frequent in hands of patients with erosive osteoarthritis. Osteoarthr Cartil 2009; 17: 1283-7.

[23] Koutroumpas AC, Alexiou IS, Vlychou M, Sakkas LI. Comparison between clinical and ultrasonographic assessment in patients with erosive osteoarthritis of the hands. Clin Rheumatol 2010; 29: 5116.

[24] Kortekaas MC, Kwok WY, Reijnierse M, Watt I, Huizinga TW, Kloppenburg M. Pain in hand osteoarthritis is associated with inflammation: the value of ultrasound. Ann Rheum Dis 2010; 69: 1367-9.

[25] Mancarella L, Magnani M, Addimanda O, Pignotti E, Galletti S, Meliconi R. Ultrasound-detected synovitis with power Doppler signal is associated with severe radiographic damage and reduced cartilage thickness in hand osteoarthritis. Osteoarthr Cartil 2010; 18: 1263-8.

[26] Schmidt WA, Schmidt H, Schicke B, Gromnica-Ihle E. Standard reference values for musculoskeletal ultrasonography. Ann Rheum Dis 2004; 63: 988-94.

[27] Arnett FC, Edworthy SM, Bloch DA, et al. The American Rheumatism Association 1987 revised criteria for the classification of rheumatoid arthritis. Arthritis Rheum 1988; 31: 315-24.

[28] Wiell C, Szkudlarek M, Hasselquist M, et al. Ultrasonography, magnetic resonance imaging, radiography, and clinical assessment of inflammatory and destructive changes in fingers and toes of patients with psoriatic arthritis. Arthritis Res Ther 2007; 9: R119.

[29] Bade H, Koebke J, Nieden A. Radiologic anatomy of the metacarpophalangeal joints II to V. Surg Radiol Anat 1997; 19: 323-7.

[30] Thiele R, Paxton L, Marston B, Tabechian D, Anandarajah A. Erosive osteoarthritis is not associated with invading synovial tissue: an ultrasound study. Arthritis Rheum [Abstract] 2010; 62: S674.

[31] Addimanda O, Mancarella L, Dolzani P, et al. Clinical and radiographic distribution of structural damage in erosive and non erosive hand osteoarthritis. Arthritis Care Res (Hoboken) 2012; 64(7): 1046-53.

This is an open access article licensed under the terms of the Creative Commons Attribution Non-Commercial License (http://creativecommons.org/licenses/by-nc/ $3.0 /$ ) which permits unrestricted, non-commercial use, distribution and reproduction in any medium, provided the work is properly cited. 